\title{
Ultrastrong coupling in two-resonator circuit QED
}

\author{
A. Baust, ${ }^{1,2,3}$ E. Hoffmann,,${ }^{1,2}$ M. Haeberlein, ${ }^{1,2}$ M. J. Schwarz, ${ }^{1,2,3}$ P. Eder, ${ }^{1,2,3}$ J. Goetz, ${ }^{1,2}$ F. Wulschner, ${ }^{1,2}$ E. Xie, ${ }^{1,2}$ \\ L. Zhong, ${ }^{1,2,3}$ F. Quijandría, ${ }^{4}$ D. Zueco, ${ }^{5,6}$ J.-J. García Ripoll, ${ }^{7}$ L. García-Álvarez, ${ }^{8}$ G. Romero, ${ }^{8,9}$ E. Solano, ${ }^{8,10}$ \\ K. G. Fedorov, ${ }^{1,2}$ E. P. Menzel, ${ }^{1,2}$ F. Deppe, $,{ }^{1,2,3},{ }^{*}$ A. Marx, ${ }^{1}$ and R. Gross ${ }^{1,2,3, \dagger}$ \\ ${ }^{1}$ Walther-Meißner-Institut, Bayerische Akademie der Wissenschaften, D-85748 Garching, Germany \\ ${ }^{2}$ Physik-Department, Technische Universität München, D-85748 Garching, Germany \\ ${ }^{3}$ Nanosystems Initiative Munich (NIM), Schellingstraße 4, 80799 München, Germany \\ ${ }^{4}$ Microtechnology and Nanoscience, MC2, Chalmers University of Technology, SE-41296 Göteborg, Sweden \\ ${ }^{5}$ Instituto de Ciencia de Materiales de Aragón and Departamento de Física de la Materia Condensada, \\ CSIC-Universidad de Zaragoza, 50009 Zaragoza, Spain \\ ${ }^{6}$ Fundación ARAID, Paseo María Agustín 36, 50004 Zaragoza, Spain \\ ${ }^{7}$ Instituto de Fisica Fundamental, IFF-CSIC, Calle Serrano 113b, Madrid E-28006, Spain \\ ${ }^{8}$ Department of Physical Chemistry, University of the Basque Country UPV/EHU, Apartado 644, E-48080 Bilbao, Spain \\ ${ }^{9}$ Departamento de Física, Universidad de Santiago de Chile (USACH), Avenida Ecuador 3493, 917-0124, Santiago, Chile \\ ${ }^{10}$ IKERBASQUE, Basque Foundation for Science, Maria Diaz de Haro 3, 48013 Bilbao, Spain \\ (Received 3 March 2015; revised manuscript received 28 April 2016; published 1 June 2016)
}

\begin{abstract}
We report on ultrastrong coupling between a superconducting flux qubit and a resonant mode of a system comprised of two superconducting coplanar stripline resonators coupled galvanically to the qubit. With a coupling strength as high as $17.5 \%$ of the mode frequency, exceeding that of previous circuit quantum electrodynamics experiments, we observe a pronounced Bloch-Siegert shift. The spectroscopic response of our multimode system reveals a clear breakdown of the Jaynes-Cummings approximation. In contrast to earlier experiments, the high coupling strength is achieved without making use of an additional inductance provided by a Josephson junction.
\end{abstract}

DOI: 10.1103/PhysRevB.93.214501

\section{INTRODUCTION}

Circuit quantum electrodynamics (QED) [1] has not only become a versatile toolbox for quantum information processing [2,3] and quantum simulation [4-6] but is also a powerful platform for the study of light-matter interaction $[7,8]$ and fundamental aspects of quantum mechanics [9-12]. In contrast to the field of cavity QED, where the interaction between a natural atom and the light field confined in a three-dimensional optical cavity is studied, the building blocks of the circuit QED architecture are superconducting quantum bits acting as artificial atoms and quasi-one-dimensional superconducting transmission line resonators with resonant frequencies in the microwave regime. Since the mode volumes of the latter are small compared to those of three-dimensional optical cavities and the dipole moments of the artificial atoms are orders of magnitude larger than those of their natural counterparts, in circuit QED setups the coupling strength between the artificial atom and quantized resonator modes can reach a significant fraction of the system energy. Remarkably, even the regime of ultrastrong coupling can be reached in superconducting circuits where the Jaynes-Cummings approximation breaks down [7]. In this situation, the interaction between light and matter can only be described correctly by the quantum Rabi model $[13,14]$ which also takes into account the counterrotating terms describing processes where the number of excitations is no longer conserved. Reaching the regime of ultrastrong coupling paves the way for various applications and the study of interesting phenomena. For instance, it allows

\footnotetext{
*frank.deppe@wmi.badw.de

†rudolf.gross@wmi.badw.de
}

for the realization of ultrafast gates [15] and provides deeper insight into Zeno physics [16] or photon transfer through cavity arrays [17]. Furthermore, a protocol allowing one to simulate the regime of ultrastrong coupling with a standard circuit QED setup has been suggested [18]. Such simulations can be used to interpret the results obtained in actual ultrastrong coupling experiments.

In this work, we demonstrate physics beyond the JaynesCummings approximation in a circuit QED architecture consisting of two coplanar stripline resonators and a superconducting flux qubit coupled galvanically to both of them. We discuss the resonant mode structure of this system and present a detailed analysis of the achieved high coupling strength. The multimode structure of our system provides an unambiguous spectroscopic proof for the breakdown of the Jaynes-Cummings approximation. Furthermore, we find that ultrastrong coupling of a qubit to a distributed resonator structure can be reached solely by the geometrical configuration of the latter without making use of additional inductive elements realized for example by Josephson junctions.

\section{SAMPLE CONFIGURATION AND MEASUREMENT SETUP}

The sample is composed of two coplanar stripline resonators, A and B, fabricated in Nb technology on a thermally oxidized Si substrate with fundamental mode frequencies $\omega_{\mathrm{R}} / 2 \pi=4.896 \mathrm{GHz}$; cf. Fig. 1(a) and Fig. 1(b). The detuning between the two resonators is found to be small and therefore disregarded. A superconducting persistent current flux qubit [19] is coupled galvanically to the signal lines of both resonators at the position of the current antinodes of the lowest frequency modes as shown in Figs. 1(c)-1(e). The flux qubit 

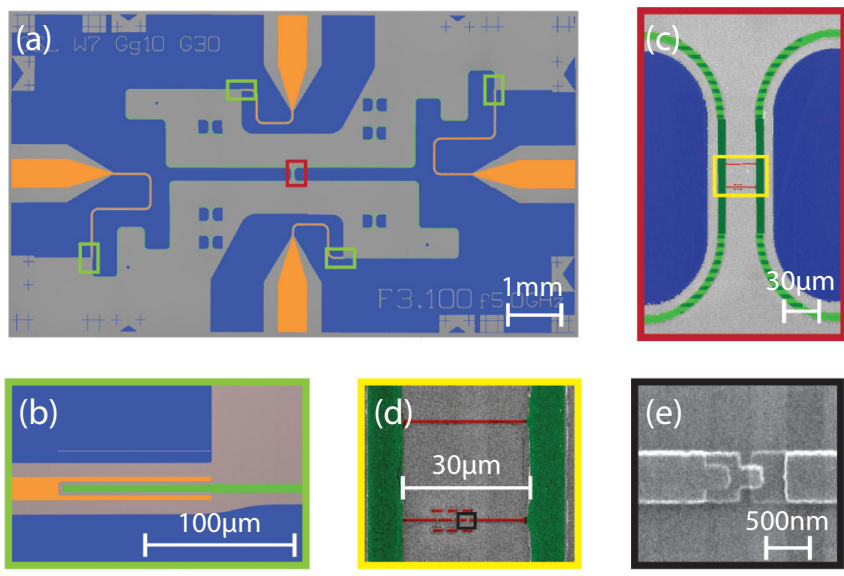

(f)

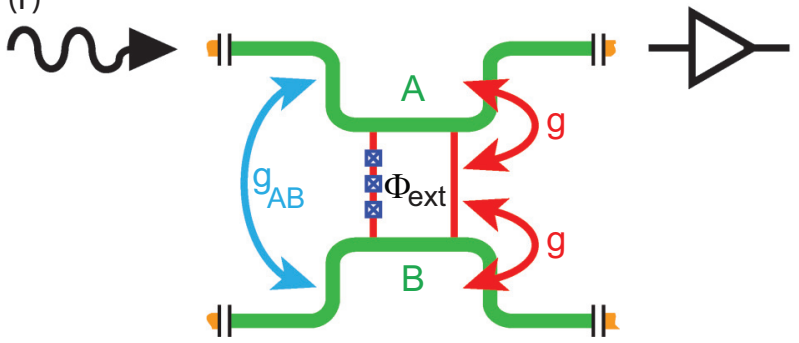

FIG. 1. Sample and measurement setup. (a) False-color image of the sample chip. $\mathrm{Nb}$ ground planes are shown in blue and feed lines in orange. The resonator signal lines reside along the ground plane edges. The green and red rectangles mark the areas shown on an enlarged scale in (b) and (c), respectively. (b) Coupling capacitor defining the resonators. (c) Resonator coupling area with signal lines (green) and flux qubit (red). Light/dark green stripes highlight Nb-Al overlap areas. The yellow rectangle marks the area shown in (d). (d) Flux qubit galvanically coupled to both resonators. The black rectangle marks the area shown in (e). (e) $\mathrm{Al} / \mathrm{AlO}_{\mathrm{x}} / \mathrm{Al}$ Josephson junction fabricated using shadow evaporation. (f) Sketch of the coupling mechanisms and measurement setup. The wiggly arrow symbolizes the input microwave line connected to resonator A and the black triangle denotes the corresponding output line featuring microwave amplifiers. The crosses intersecting one qubit branch symbolize the three Josephson junctions.

consists of a superconducting Al loop intersected by three Josephson junctions. Two of them have the critical current $I_{\mathrm{c}}$ and the phase drops across them are denoted by $\phi_{1}$ and $\phi_{2}$. The third one has a junction area smaller by a factor $\alpha \simeq 0.7$. We mount the sample inside a gold-plated copper box attached to the base temperature stage of a dilution refrigerator stabilized at $45 \mathrm{mK}$. The magnetic flux $\Phi_{\text {ext }}$ applied to the qubit can be adjusted by means of a superconducting solenoid mounted on top of the sample box. Further technical details on the fabrication process and device parameters can be found in Ref. [20] and Ref. [21]. Since some of the nomenclature used in the present work was introduced in previous work on this sample [21], we briefly reiterate the main findings here. As discussed in Ref. [21], the qubit can be used to tune and switch the coupling between the two resonators. In addition to the geometric coupling $g_{\mathrm{AB}} / 2 \pi=8.4 \mathrm{MHz}$ there is the qubit mediated second-order dynamic coupling which depends on the magnetic flux applied to the qubit loop and on the qubit state. If the qubit is in the ground state, there exist certain flux values which we refer to as switch setting conditions, where the geometric coupling is (in the ideal case) fully compensated by the dynamical coupling such that the total coupling between the two resonators vanishes [22]. Conversely, when the qubit is saturated by means of a strong excitation signal, the dynamical coupling is zero regardless of the flux applied to the qubit loop and the total coupling between the two resonators becomes $g_{\mathrm{AB}}$. The dependence of the dynamical coupling on the qubit state can be used to realize switchable coupling between the two resonators. As demonstrated in Ref. [21], setting the flux operation point to a switch setting condition and applying a drive pulse to the qubit allows one to switch the coupling between the resonators A and B to the desired value between zero and $g_{\mathrm{AB}}$ depending on the drive pulse amplitude. This tunable coupler physics involves only two particular modes of the device. However, as we discuss in the following, the nature of the galvanic qubit-resonator coupling implies a more complex mode structure.

\section{MODE STRUCTURE}

We first probe the coupled qubit-resonator system by measuring the transmission through resonator A depending on the magnetic flux applied to the qubit loop; cf. Fig. 1(f). For the measurement, the qubit is kept in the ground state and the input power is chosen such that the mean resonator population is approximately one photon on average. For coupled microwave resonators, we expect to observe two resonant modes corresponding to out-of-phase and in-phase oscillating currents in the two resonators; cf. Fig. 2(a) and Fig. 2(b). Following the nomenclature in Ref. [21], we refer to
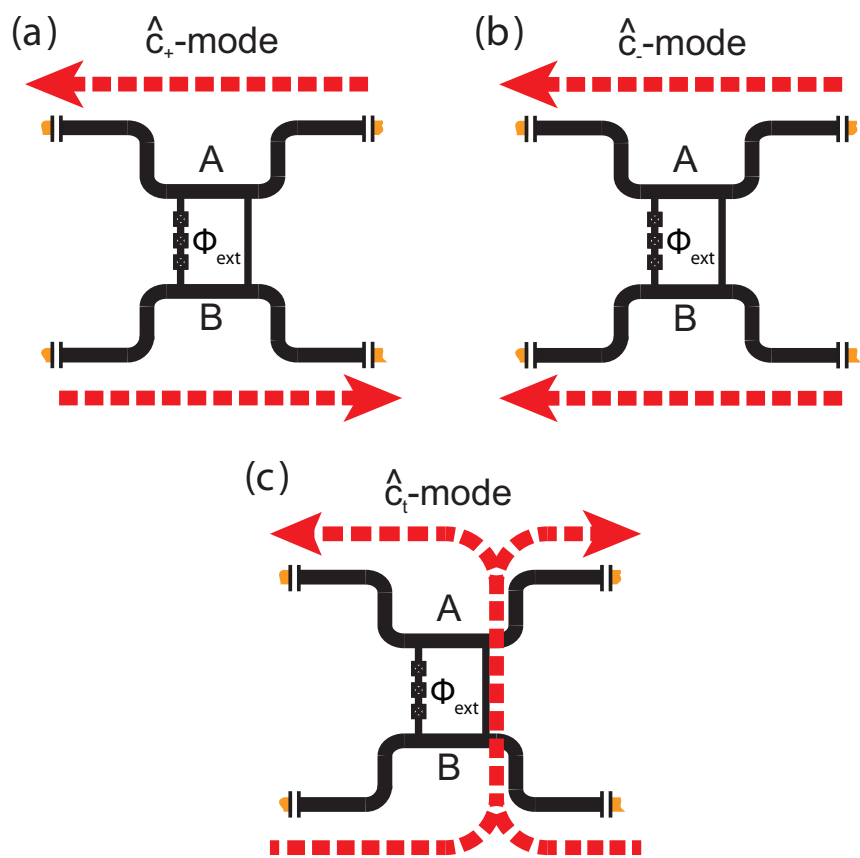

FIG. 2. Resonant modes of the galvanically coupled qubitresonator system. The arrows indicate in-phase and out-of-phase oscillating currents. (a) Antiparallel mode. (b) Parallel mode. (c) Transverse mode. 
(a)

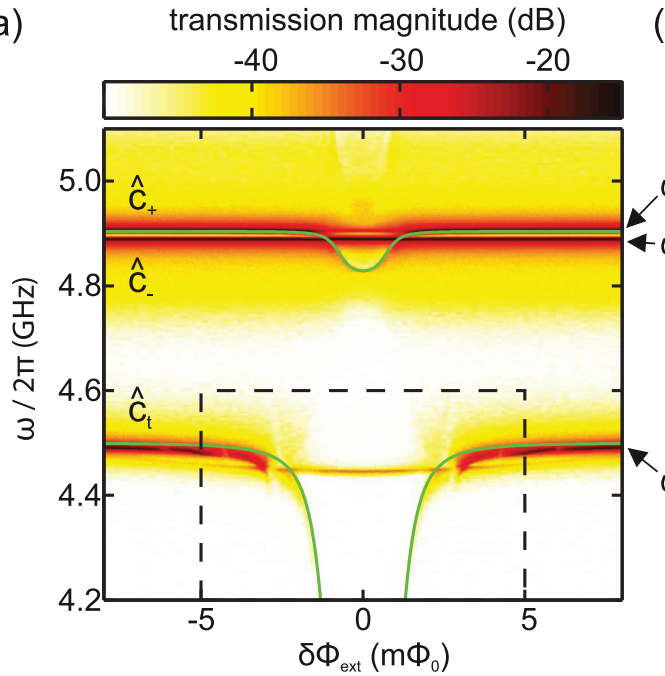

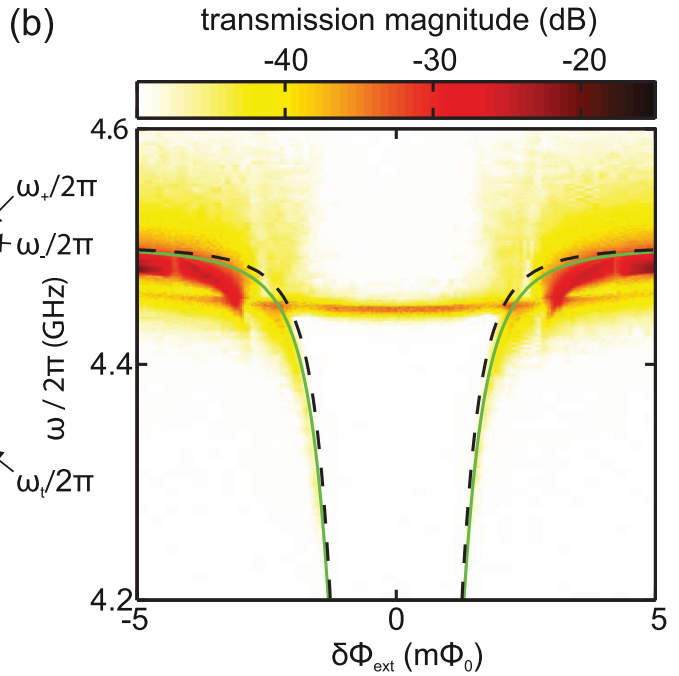

FIG. 3. (a) Transmission measured through resonator A as a function of the applied magnetic flux with the qubit in the ground state. Green line: fit using the Hamiltonian of Eq. (1). The area shown in panel (b) is marked by the black rectangle. (b) Detail of (a). Solid green line: fit using the Hamiltonian of Eq. (1). Dashed black line: Jaynes-Cumming approximation obtained using the parameters producing the green line but neglecting the counter-rotating terms in the Hamiltonian of Eq. (1).

these modes as the antiparallel and parallel mode and assign to them the annihilation operators $\hat{c}_{+}$and $\hat{c}_{-}$, respectively. They can be identified in the spectroscopy data presented in Fig. 3(a). Far away from the qubit degeneracy point $\delta \Phi_{\text {ext }} \equiv$ $\Phi_{\text {ext }}-\Phi_{0} / 2=0$, where $\Phi_{\text {ext }}$ is the external magnetic flux and $\Phi_{0}$ is the flux quantum, the dynamical coupling is negligible and the resonant frequencies of the antiparallel and parallel mode are given by $\omega_{+} / 2 \pi=\left(\omega_{\mathrm{R}}+g_{\mathrm{AB}}\right) / 2 \pi=4.904 \mathrm{GHz}$ and $\omega_{-} / 2 \pi=\left(\omega_{\mathrm{R}}-g_{\mathrm{AB}}\right) / 2 \pi=4.888 \mathrm{GHz}$.

However, the galvanic coupling of the qubit to both resonators gives rise to a third mode $\hat{c}_{\mathrm{t}}$ which we refer to as the "transverse mode." It is identified as a parallel mode across the qubit as shown in Fig. 2(c). Far away from the qubit degeneracy point, its resonant frequency is found to be $\omega_{\mathrm{t}} / 2 \pi=4.508 \mathrm{GHz}$. To explain the large frequency detuning between the transverse and the (anti)parallel mode, we assume that the inductance of the qubit has to be taken into account in order to correctly describe the frequency of the transverse mode. Following Ref. [23] and Ref. [24], we calculate the resonant frequency of the transverse mode to $\omega_{\mathrm{t}}=\omega_{\mathrm{R}} /(1+$ $L_{\mathrm{Q}} / L_{\mathrm{R}}$ ), where $L_{\mathrm{Q}}$ is the inductance of the flux qubit and $L_{\mathrm{R}}$ is the inductance of a single resonator. The latter is given by $L_{\mathrm{R}}=Z / 2 \omega_{\mathrm{R}}=8.2 \mathrm{nH}$, where $Z=80 \Omega$ is the characteristic impedance of the resonator [25]. The inductance of the flux qubit is given by $L_{\mathrm{Q}}=\left(\partial^{2} U_{\mathrm{Q}} / \partial \Phi_{\text {ext }}^{2}\right)^{-1}$, where $U_{\mathrm{Q}}=$ $E_{\mathrm{J}}\left[2+\alpha-\cos \phi_{1}-\cos \phi_{2}-\alpha \cos \left(2 \pi f+\phi_{1}-\phi_{2}\right)\right]$ is the flux qubit potential [26], $f=\Phi_{\text {ext }} / \Phi_{0}$ is the frustration and $E_{\mathrm{J}}=\Phi_{0} I_{\mathrm{c}} / 2 \pi$ is the Josephson energy. Introducing $\phi_{-} \equiv\left(\phi_{1}-\phi_{2}\right) / 2$, the inductance of the flux qubit reads as $L_{\mathrm{Q}}=\Phi_{0} /\left[2 \pi \alpha I_{\mathrm{c}} \cos \left(2 \pi f+2 \phi_{-}\right)\right]$with a minimum value of $L_{\mathrm{Q}}\left(f=0, \phi_{-}=0\right)=\Phi_{0} / 2 \pi \alpha I_{\mathrm{c}}=719 \mathrm{pH}$, yielding a resonant frequency of $\omega_{\mathrm{t}, \text { theo }} / 2 \pi=4.501 \mathrm{GHz}$. This value is in excellent agreement with the experimental value $\omega_{\mathrm{t}} / 2 \pi=4.508 \mathrm{GHz}$ measured far away from the degeneracy point.

Based on the above discussion, our circuit is naturally represented by the Hamiltonian describing a dipolar interaction of the qubit with the relevant resonant modes,

$$
\begin{aligned}
\hat{H}= & \hat{H}_{\mathrm{Q}}+\sum_{\substack{n=\\
\{t,+, 3 t, 3+\}}} \hat{H}_{n}+\hbar g \sqrt{2} \hat{\sigma}_{z}\left(\hat{c}_{+}^{\dagger}+\hat{c}_{+}\right) \\
& +\hbar g_{\mathrm{t}} \hat{\sigma}_{z}\left(\hat{c}_{\mathrm{t}}^{\dagger}+\hat{c}_{\mathrm{t}}\right)+\hbar g_{3 \mathrm{t}} \hat{\sigma}_{z}\left(\hat{c}_{3 \mathrm{t}}^{\dagger}+\hat{c}_{3 \mathrm{t}}\right) \\
& +\hbar g_{3+} \hat{\sigma}_{z}\left(\hat{c}_{3+}^{\dagger}+\hat{c}_{3+}\right) .
\end{aligned}
$$

Here, we are allowed to omit the $\hat{c}_{-}$mode because it does not generate a net magnetic field in the qubit loop and, hence, does not couple to the qubit. The term $\hat{H}_{\mathrm{Q}} \equiv(\varepsilon / 2) \hat{\sigma}_{z}+$ $(\Delta / 2) \hat{\sigma}_{x}$ is the qubit Hamiltonian and $\hat{H}_{n} \equiv \hbar \omega_{n} \hat{c}_{n}^{\dagger} \hat{c}_{n}$ is the Hamiltonian describing the resonant mode $\hat{c}_{n}$. The quantity $\Delta$ is the qubit energy gap, $\epsilon\left(\Phi_{\text {ext }}\right)=2 I_{\mathrm{p}} \delta \Phi_{\text {ext }}$ denotes the qubit energy bias, and $I_{\mathrm{p}}=I_{\mathrm{c}} \sqrt{1-(2 \alpha)^{-2}}$ the qubit persistent current. $\hat{\sigma}_{x}$ and $\hat{\sigma}_{z}$ are the Pauli operators. As shown in Ref. [21], the coupling of the qubit to the antiparallel mode is given by $g_{+}=\sqrt{2} g$. To increase precision of our description, we also take into account the third harmonic of the $\hat{c}_{\mathrm{t}}$ mode (denoted by $\hat{c}_{3 \mathrm{t}}$, located at $\left.\omega_{3 \mathrm{t}} / 2 \pi=13.1 \mathrm{GHz}\right)$ and the third harmonic of the $\hat{c}_{+}$mode (denoted by $\hat{c}_{3+}$, at $\omega_{3+} / 2 \pi=14.3 \mathrm{GHz}$ ). We do not consider the second harmonics since they exhibit current nodes at the qubit position and therefore do not couple to the qubit. The coupling strengths $g_{3 t}$ and $g_{3+}$ are not considered as independent parameters, but are calculated via $g_{3 t} / 2 \pi=$ $\left(g_{\mathrm{t}} / 2 \pi\right) \sqrt{\omega_{3 \mathrm{t}} / \omega_{\mathrm{t}}}$ and $g_{3+} / 2 \pi=\left(g_{+} / 2 \pi\right) \sqrt{\omega_{3+} / \omega_{+}}$, taking into account the current distribution in the resonator. To determine the qubit parameters and the qubit-mode coupling strengths, we diagonalize the Hamiltonian of Eq. (1) numerically and fit the resulting energy levels to the data shown in Fig. 3(a) and Fig. 3(b) (see the Appendix for fit details). In this way, we obtain a qubit energy gap $\Delta / h=3.51 \mathrm{GHz}$ and a persistent current $I_{\mathrm{p}}=469 \mathrm{nA}$. We find that the coupling strength between the qubit and each resonator is given by $g / 2 \pi=96.7 \mathrm{MHz}$ and the coupling strength of the mode $\hat{c}_{\mathrm{t}}$ to the qubit is $g_{\mathrm{t}} / 2 \pi=(787 \pm 51) \mathrm{MHz}$, which is as high 
as $17.5 \%$ of the respective mode frequency. Remarkably, this coupling strength even exceeds the relative coupling strengths observed in Ref. [7] although the coupling is determined solely by the geometrical properties of the qubit arm and not by an additional inductive element such as a Josephson junction introduced in Ref. [7] to enhance the coupling strength. To understand the origin of the exceptionally large coupling strength, we assume that the coupling strength of the qubit to resonator A and $\mathrm{B}$, respectively, is determined by the shared arms between the qubit and the resonators $\mathrm{A}$ and $\mathrm{B}$, respectively. We further assume that the transverse mode current is flowing predominantly through the qubit arm without Josephson junctions as shown in Fig. 2(c). This assumption is well justified since the geometrical inductance of the qubit arm without Josephson junctions is much smaller than the total inductance of the branch containing the three Josephson junctions. Following Ref. [27], we can estimate the geometric inductance of the qubit branch connecting the two resonators A and B (length $30 \mu \mathrm{m}$, width $0.5 \mu \mathrm{m}$, and thickness $0.1 \mu \mathrm{m}$ ) at $31 \mathrm{pH}$, which adds to the kinetic inductance [28,29] of $\sim 27 \mathrm{pH}$, yielding a total inductance of the qubit branch $L_{\mathrm{t}}=58 \mathrm{pH}$. We can further estimate the inductance of the shared arms (length $20 \mu \mathrm{m}$ ) between the resonators (total length $11.55 \mathrm{~mm}$ ) A and B and the qubit at $L_{\mathrm{r}}=L_{\mathrm{R}} \times 20 \mu \mathrm{m} / 11.55 \mathrm{~mm}=14.2 \mathrm{pH}$. The coupling strength between the antiparallel mode $\hat{c}_{3+}$ and the flux qubit is $g_{+}=\sqrt{2} g$. The total coupling strength $g_{\mathrm{t}}$ of the transverse mode $\hat{c}_{\mathrm{t}}$ to the qubit has two contributions. The first one is the coupling mediated by the shared branches between qubit and resonator and the second one is the coupling mediated by the qubit branch connecting the two resonators. Therefore, we can calculate the ratio $g_{\mathrm{t}} / g_{+}=\left(2 L_{\mathrm{r}} I_{\mathrm{p}} I_{\mathrm{R}}+\right.$ $\left.\sqrt{2} L_{\mathrm{t}} I_{\mathrm{p}} I_{\mathrm{R}}\right) /\left(L_{\mathrm{r}} I_{\mathrm{p}} I_{\mathrm{R}}\right)=\sqrt{2}+L_{\mathrm{t}} / L_{\mathrm{r}} \simeq 5.5$, where $I_{\mathrm{R}}$ is the vacuum current of each resonator. This value is in good agreement with the experimentally found ratio $g_{t} / g_{+} \simeq 5.8$.

\section{ULTRASTRONG COUPLING}

In what follows, we discuss the theoretical framework needed to describe the interaction between the qubit and the multimode structure arising from the two-resonator circuit QED architecture. First, we rotate the Hamiltonian of Eq. (1) into the qubit eigenbasis using the transformations $\hat{\sigma}_{\mathrm{z}} \rightarrow$ $\cos \theta \hat{\sigma}_{\mathrm{z}}-\sin \theta \hat{\sigma}_{\mathrm{x}}$ and $\hat{\sigma}_{\mathrm{x}} \rightarrow \sin \theta \hat{\sigma}_{\mathrm{z}}+\cos \theta \hat{\sigma}_{\mathrm{x}}$, where $\sin \theta \equiv$ $\Delta / \hbar \omega_{\mathrm{q}}$ and $\cos \theta \equiv \epsilon / \hbar \omega_{\mathrm{q}}$ and $\hbar \omega_{\mathrm{q}}=\sqrt{\Delta^{2}+\epsilon^{2}}$ is the fluxdependent qubit transition energy. In the qubit eigenbasis, the Hamiltonian reads

$$
\hat{H}^{*}=\hat{H}_{\mathrm{Q}}^{*}+\sum_{\substack{n=\\\{t,+, 3 t, 3+\}}}\left[\hat{H}_{n}+\hbar g_{n}\left(\hat{c}_{n}^{\dagger}+\hat{c}_{n}\right)\left(\cos \theta \hat{\sigma}_{\mathrm{z}}-\sin \theta \hat{\sigma}_{\mathrm{x}}\right)\right],
$$

with $\hat{H}_{\mathrm{Q}}^{*}=\frac{\hbar \omega_{\mathrm{q}}}{2} \hat{\sigma}_{\mathrm{z}}$. We note that this Hamiltonian is equivalent to the Hamiltonian of Eq. (1). At $\Phi_{\text {ext }}=\Phi_{0} / 2$, the Hamiltonian of Eq. (2) represents a multimode quantum Rabi model. Defining the qubit state raising and lowering operators $\hat{\sigma}_{ \pm} \equiv$ $\left(\hat{\sigma}_{x} \pm i \hat{\sigma}_{y}\right) / 2$, we find that the Hamiltonian of Eq. (2) explicitly contains counter-rotating terms of the form $\hat{c}_{n}^{\dagger} \hat{\sigma}_{+}$and $\hat{c}_{n} \hat{\sigma}_{-}$. For $g_{n} \ll \omega_{n}$, a rotating wave approximation reduces the Hamiltonian of Eq. (2) to the well known multimode Jaynes-Cummings
Hamiltonian for arbitrary $\Phi_{\text {ext }}$. We emphasize that the quantum Rabi model correctly describes the dipolar interaction of the qubit circuit with the resonant modes. The question arising in this context is whether the approximation of the quantum Rabi model by the Jaynes-Cumming Hamiltonian is valid for a given experiment. Only if our best effort to describe the interaction between qubit and light fields with a Jaynes-Cummings approximation yields clear deviations from the experimental data, one can claim to have reached the ultrastrong coupling regime [7]. Typically, $g / \omega \simeq 10 \%-20 \%$ is required for this task. Despite the breakdown of the Jaynes-Cummings approximation, the system dynamics may still reflect the intuition of several distinct, but coupled systems exchanging excitations. This intuition breaks down completely in the deep strong coupling regime [30], where $g \gtrsim \omega$ and the dynamics of the system is characterized by the emergence of two parity chains.

\section{BREAKDOWN OF THE JAYNES-CUMMINGS APPROXIMATION}

In this section, we discuss that our multipartite circuit QED setup comprised of a flux qubit galvanically coupled to two resonators cannot be described within a Jaynes-Cummings approximation. Instead, it has to be treated within the more general quantum Rabi model.

To this end, we first revisit our fit of the Hamiltonian of Eq. (1) to the single-tone transmission spectroscopy data used to characterize our device in Sec. III. Always using the same set of parameters obtained from this fit, we compare the energy levels from the quantum Rabi model represented by the Hamiltonian of Eq. (1) to the Jaynes-Cummings approximation obtained by dropping the counter-rotating terms from this Hamiltonian. For the transverse mode $\hat{c}_{t}$, we see in Fig. 3(b) that there is only a relatively small quantitative difference in the form of a quantum Bloch-Siegert shift [8] between the two scenarios. Such a Bloch-Siegert shift is proportional to $g^{2} \sin ^{2} \theta /\left(\omega_{\mathrm{q}}+\omega_{\mathrm{r}}\right)$ and, hence, produces a pronounced effect on the measured spectra only very close to the qubit degeneracy point for $\left|\delta \Phi_{\mathrm{x}}\right| \lesssim 1 \mathrm{~m} \Phi_{0}$. In contrast to the $\hat{c}_{\mathrm{t}}$ mode, a significant qualitative difference between quantum Rabi model and Jaynes-Cummings approximation can be observed for the $\hat{c}_{+}$mode as shown in Fig. 4(a). Here, quantum Rabi model (green line) and experimental data agree very well, while neglecting the counter-rotating terms (dashed black line) in the Hamiltonian of Eq. (1) produces a clear qualitative deviation with respect to the data.

So far, our analysis is based on the structure of our circuit, which naturally suggests that the transverse mode occurs because of the galvanic coupling. However, one may simply ignore all supportive evidence presented so far for this argument and claim otherwise. In other words, one may simply postulate that the source of ultrastrong coupling in the circuit, the transverse mode $\hat{c}_{\mathrm{t}}$, should be treated as an independent phenomenon and, thus, omitted from the Hamiltonian of Eq. (1). Under this assumption, one could fit only the coupler modes to the Hamiltonian

$$
\begin{aligned}
\hat{H}= & \hat{H}_{\mathrm{Q}}^{*}+\sum_{\mathrm{n}=\{+, 3+\}} \hat{H}_{n}+\hbar g_{+}\left(\hat{c}_{+}^{\dagger} \hat{\sigma}_{-}+\hat{c}_{+} \hat{\sigma}_{+}\right) \\
& +\hbar g_{3+}\left(\hat{c}_{3+}^{\dagger} \hat{\sigma}_{-}+\hat{c}_{3+} \hat{\sigma}_{+}\right),
\end{aligned}
$$



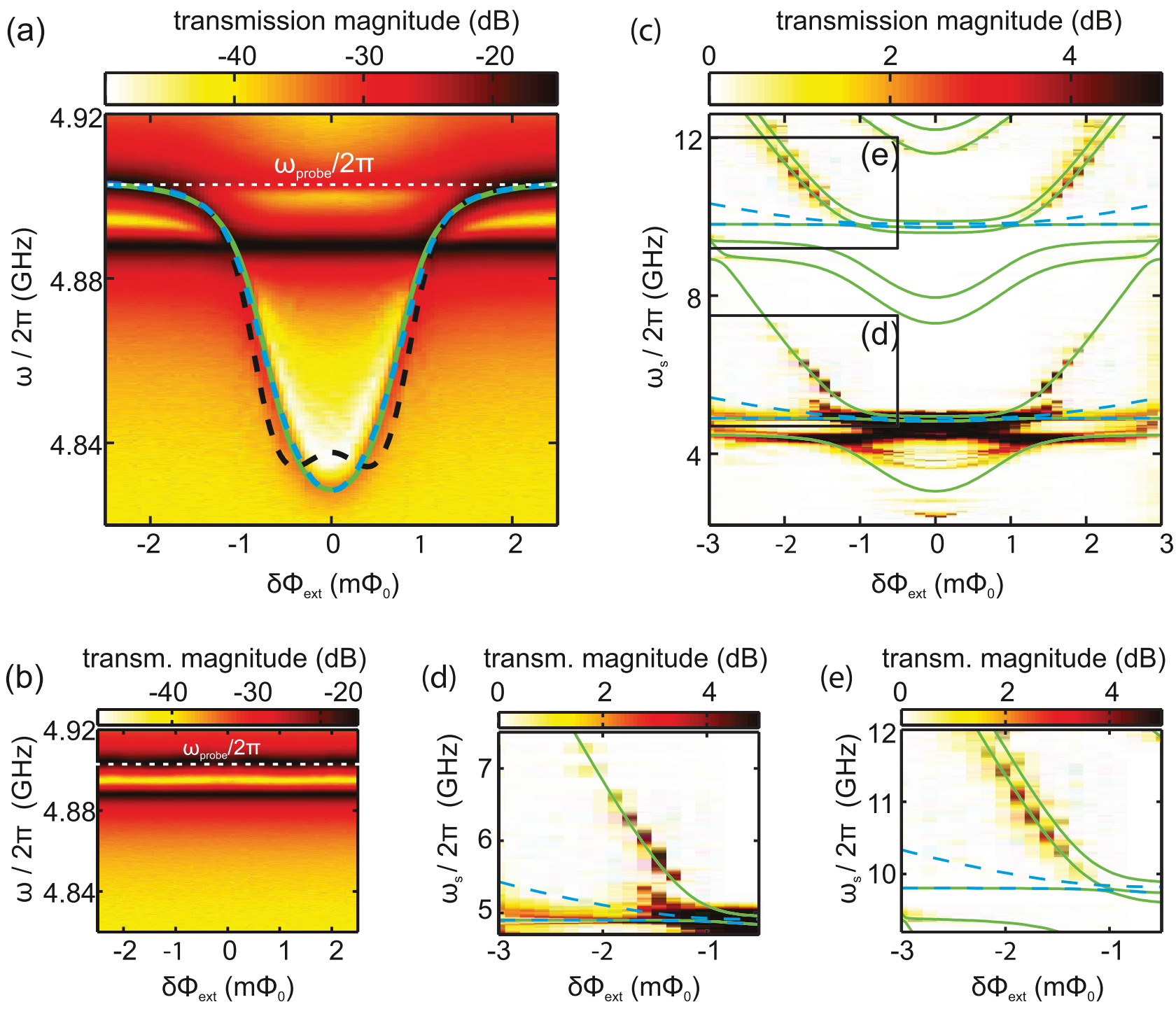

FIG. 4. Breakdown of the Jaynes-Cummings approximation. (a) Transmission measured through resonator A as a function of the magnetic flux applied to the flux qubit with the qubit in the ground state. Data: detail from Fig. 3(a). Green line: fit using the quantum Rabi Hamiltonian of Eq. (1). Dashed black line: Jaynes-Cumming approximation using the parameters producing the green line but neglecting the counter-rotating terms in the Hamiltonian of Eq. (1). Dashed blue line: fit using the Jaynes-Cummings Hamiltonian of Eq. (3), where the transverse mode is treated as an independent phenomenon. Dashed white line: probe frequency $\omega_{\text {probe }}$ used for two-tone spectroscopy. (b) Same as (a), but with the qubit driven by a strong microwave tone. (c) Two-tone spectroscopy. Green and dashed blue lines as in panel (a). $\omega_{\mathrm{s}}$ is the variable spectroscopy tone. Color code: transmission magnitude measured at $\omega_{\text {probe }}$ (d),(e) Details from (c).

which contains no counter-rotating terms anymore. As shown in Fig. 4(a), the $\hat{c}_{+}$transmission data are described well by this ansatz. However, the resulting qubit parameters deviate strongly from those obtained in Sec. III by fitting the Hamiltonian of Eq. (1) to the data. In order to verify which of the two parameter sets (and thus Hamiltonians) is appropriate, we employ an additional, independent measurement technique: two-tone spectroscopy of the qubit. To this end, we record the transmission through resonator $\mathrm{A}$ at the frequency of $\omega_{\text {probe }} / 2 \pi=4.904 \mathrm{GHz}$. When the qubit is far detuned, $\omega_{\text {probe }}$ corresponds to the resonant frequency of the $\hat{c}_{+}$mode. In addition, a second microwave tone, the spectroscopy tone with variable frequency $\omega_{\mathrm{s}}$ is applied to the coupled qubit-resonator system via the input port of resonator B. When the qubit is in the ground state, the measured transmission as a function of the magnetic flux applied to the qubit loop corresponds to a cut through Fig. 4(a) along $\omega_{+} / 2 \pi$ as highlighted by the dashed white line. When the qubit is saturated by means of the spectroscopy tone, the qubit state is described by the density matrix $\rho_{\mathrm{M}}=\frac{1}{2}(|g\rangle\langle g|+| e\rangle\langle e|)$ and the transmission spectrum turns into the one shown in Fig. 4(b). Evidently, the transmission magnitude at $\omega_{\text {probe }}$ increases near the degeneracy point when the qubit is driven.

Using this protocol, we record the change in resonator transmission as a function of the spectroscopy tone frequency $\omega_{\mathrm{s}}$ and the applied magnetic flux. The results are displayed in Figs. 4(c)-4(e). We first compare the measured data to the energy level spectrum of the Hamiltonian of Eq. (2) by calculating the energy differences between the ground state and the 15 lowest energy levels. As it can be seen, there is 
very good agreement between the two-tone spectroscopy data and their description within the full Hamiltonian of Eq. (2). The absence of spectroscopic response for the energy levels near $2 \omega_{+}$and $2 \omega_{\mathrm{t}}$ in Fig. $4(\mathrm{c})$ is due to the fact that the second harmonics of the $\hat{c}_{+}$and $\hat{c}_{\mathrm{t}}$ modes exhibit current nodes at the position of the qubit and therefore do not couple to it.

Next, we analyze the energy level spectrum calculated from the qubit parameters found by a fit using only the $\hat{c}_{+}$mode in the Jaynes-Cummings approximation, i.e., Eq. (3). Here, we find clear deviations from the qubit two-tone spectroscopy data. This effect becomes most apparent in Fig. 4(c) and Fig. 4(d), where the dashed blue line produced by Eq. (3) fails to explain the dips of the qubit hyperbola. In contrast, these dips are captured by the upper green line produced by the quantum Rabi model including the transverse mode. The slope of this upper green line for $\delta \Phi_{\text {ext }} \lesssim-1.3 \mathrm{~m} \Phi_{0}$ is, up to fundamental constants, proportional to the qubit critical current $I_{\mathrm{p}}$. In other words, the two-tone experiments provide an independent mean to confirm the interpretation of the transverse mode derived from the circuit geometry in Fig. 2. As discussed before and shown in Fig. 4(a), in this situation the Jaynes-Cummings approximation is not compatible with the single-tone transmission data. Hence we have successfully demonstrated the ultrastrong coupling regime because our data can only be explained when taking the counter-rotating terms of the transverse mode into account.

Finally, we compare our findings to previous work on ultrastrong coupling in superconducting circuits. In the present sample, the access to both resonator and qubit spectroscopy data allows us to rigorously rule out the validity of the Jaynes-Cummings approximation without having to assume the validity of the quantum Rabi model. Hence our analysis goes beyond the treatment presented in Ref. [8]. In addition, the present work is markedly different from the approach used in Ref. [7]. There, it was shown that in a multimode system the number of excitations is no longer preserved in the ultrastrong coupling regime. Despite this difference, it appears that physics beyond the Jaynes-Cummings approximation in circuit QED is favorably demonstrated by analyzing the complex mode structure of multipartite setups.

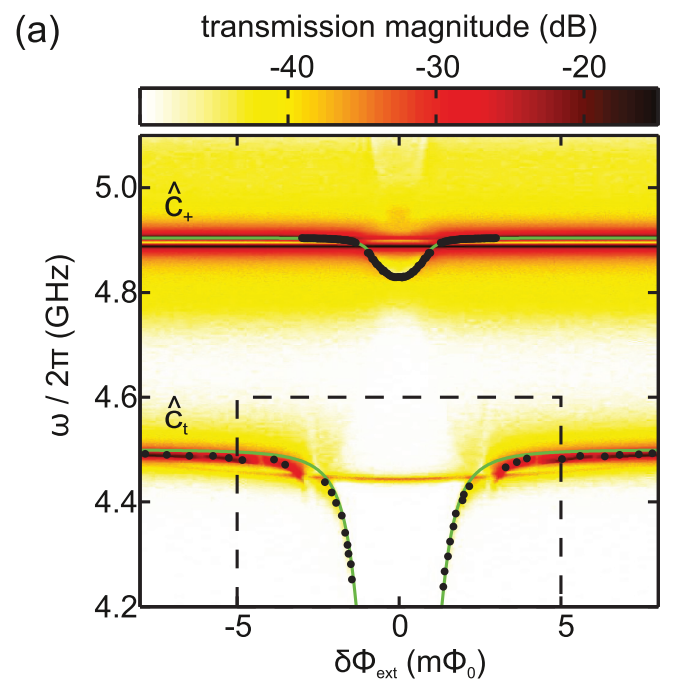

\section{CONCLUSIONS}

In conclusion, we demonstrate the breakdown of the Jaynes-Cummings approximation in a system comprised of two coplanar stripline resonators and a persistent current flux qubit coupled galvanically to both of them. We analyze the complex mode structure and find that the coupling of one resonant mode to the qubit reaches $17 \%$ of the mode frequency, exceeding that of previous circuit QED experiments $[7,8]$. We show that both the mode frequency and the coupling strength are in good agreement with theoretical calculations based on the quantum Rabi model. Analyzing the resonator and qubit spectroscopy data clearly shows that the Jaynes-Cummings approximation no longer provides an appropriate description of the observed behavior, confirming that our circuit QED setup is in the regime of ultrastrong coupling. In the sample, a remarkably large coupling strength is reached without utilizing the inductance of an additional Josephson junction. As a future perspective, combining different methods for enhancing the coupling strength may provide access to the regime of deep strong coupling [30], giving experimental insight into a completely novel regime of light-matter interaction.

\section{ACKNOWLEDGMENTS}

This work is supported by the German Research Foundation through SFB 631 and FE 1564/1-1; Spanish MINECO FIS2012-36673-03-02, MAT2014-53432-C5-1-R, FIS201455867-P and FIS2012-33022; CAM Research Network QUITEMAD+; UPV/EHU UFI 11/55, UPV/EHU PhD Grant, and Basque Government IT472-10; the Fondo Nacional de Desarrollo Cientifico y Tecnológico (FONDECYT, Chile) under Grant 1150653; the EU projects CCQED, PROMISCE, and SCALEQIT. We further acknowledge GEFENOL.

\section{APPENDIX}

In this section, we first provide technical details on how we obtain the fit lines in Fig. 3(a) and Fig. 3(b). We employ

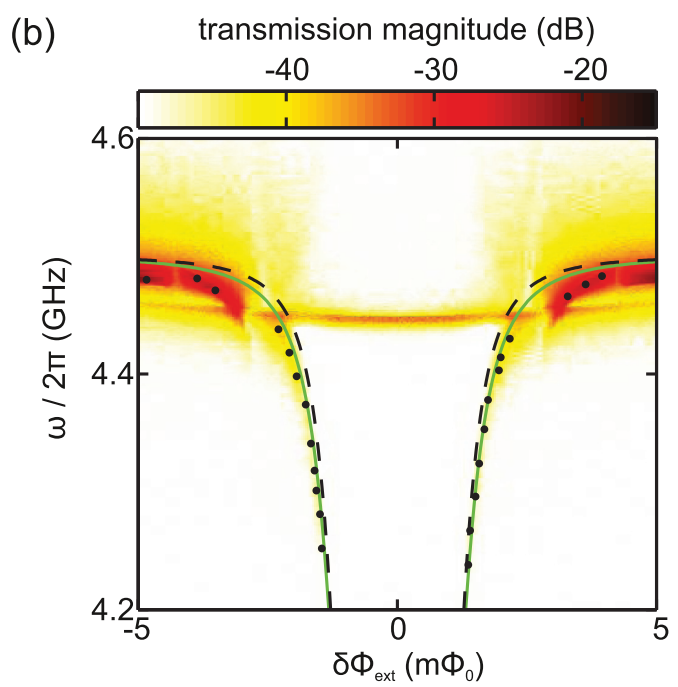

FIG. 5. Panels from Fig. 3(a) and Fig. 3(b) including the data points (black dots) used for the fit. 
a simplex algorithm. The mode frequencies $\omega_{n}$ are not used as fit parameters, but are determined from the transmission spectrum at $\delta \Phi_{\text {ext }}=\Phi_{0} / 2$. We use 118 data points of the $\hat{c}_{+}$ mode and 35 data points of the $\hat{c}_{\mathrm{t}}$ mode. These data points are shown in Fig. 5(a) and Fig. 5(b). They correspond to peaks in the transmission spectra. As the $\hat{c}_{+}$mode extends over a considerably smaller frequency span than the $\hat{c}_{\mathrm{t}}$ mode, we consider a larger number of data points for the $\hat{c}_{+}$mode. As a consequence, both modes contribute approximately equally to the statistical fit error. In order to ensure robustness of the fitting algorithm, we repeat the fit 100 times based on random subsets of 30 data points. In this way, we find a standard deviation of $51 \mathrm{MHz}$ for $g_{\mathrm{t}}$.

Next, we discuss resonant structures in our data not directly relevant for the ultrastrong coupling discussion in the main text. First, we briefly discuss the origin of the resonant structure which is visible near the degeneracy point at the frequency of $4.904 \mathrm{GHz}$; cf. Fig. 6. We find good agreement between this resonant structure and the transition between the eigenstates corresponding to the second and the sixth lowest eigenenergies of the Hamiltonian of Eq. (1). Compared to the antiparallel mode, the additional resonant structure is suppressed by $\sim 14 \mathrm{~dB}$. Hence this structure might arise from a small finite population of the second energy level due to the finite sample temperature of $45 \mathrm{mK}$ and the very large coupling strength of the qubit to the transverse mode. This interpretation is also in agreement with a similar resonant structure observed in Ref. [8].

In contrast to this feature, the faint resonance with weak flux dependence visible at a frequency of approximately $4.44 \mathrm{GHz}$ in Fig. 3 is not captured by our analysis. However, preliminary

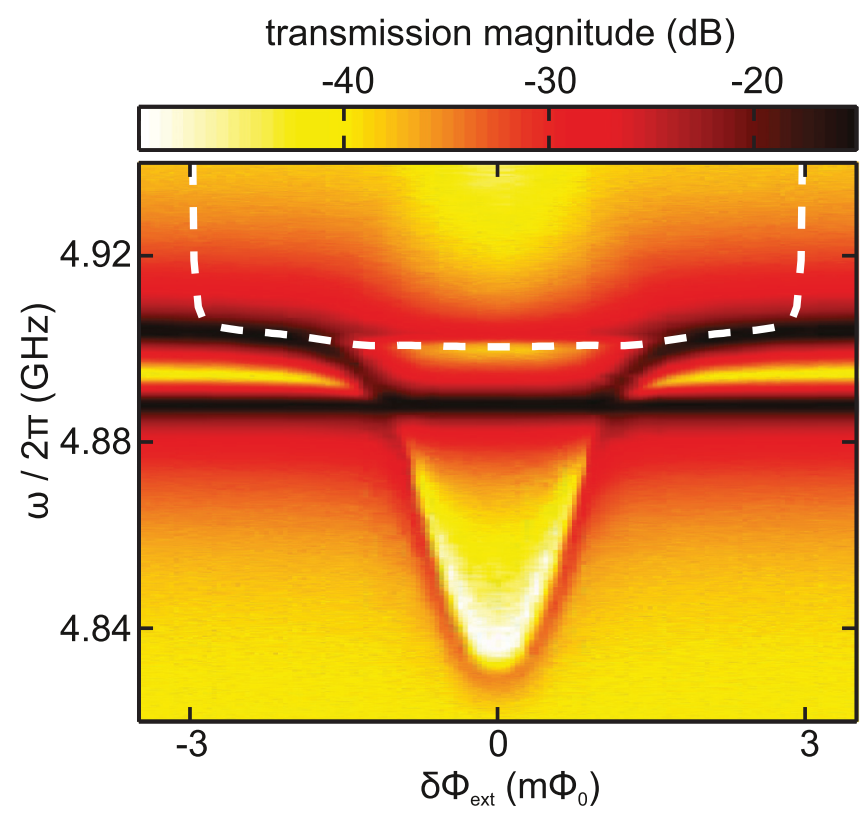

FIG. 6. Transmission measured through resonator A depending on the magnetic flux applied to the flux qubit. Dashed white line: transition between eigenstates of the Hamiltonian of Eq. (1) as described in the main text.

numerical simulations suggest that this resonance might be caused by thermally excited multiphoton transitions similar to the ones observed in Refs. [31-33] and, hence, has no impact on the main conclusions of this work.
[1] A. Wallraff, D. I. Schuster, A. Blais, L. Frunzio, R.-S. Huang, J. Majer, S. Kumar, S. M. Girvin, and R. J. Schoelkopf, Nature (London) 431, 162 (2004).

[2] M. Pechal, L. Huthmacher, C. Eichler, S. Zeytinoğlu, A. A. Abdumalikov, Jr., S. Berger, A. Wallraff, and S. Filipp, Phys. Rev. X 4, 041010 (2014).

[3] Y. Chen, C. Neill, P. Roushan, N. Leung, M. Fang, R. Barends, J. Kelly, B. Campbell, Z. Chen, B. Chiaro, A. Dunsworth, E. Jeffrey, A. Megrant, J. Y. Mutus, P. J. J. O’Malley, C. M. Quintana, D. Sank, A. Vainsencher, J. Wenner, T. C. White, M. R. Geller, A. N. Cleland, and J. M. Martinis, Phys. Rev. Lett. 113, 220502 (2014).

[4] A. A. Houck, H. E. Tureci, and J. Koch, Nat. Phys. 8, 292 (2012).

[5] J. Raftery, D. Sadri, S. Schmidt, H. E. Türeci, and A. A. Houck, Phys. Rev. X 4, 031043 (2014).

[6] Y. Chen, P. Roushan, D. Sank, C. Neill, E. Lucero, M. Mariantoni, R. Barends, B. Chiaro, J. Kelly, A. Megrant, J. Y. Mutus, P. J. J. O’Malley, A. Vainsencher, J. Wenner, T. C. White, Y. Yin, A. N. Cleland, and J. M. Martinis, Nat. Commun. 5, 5184 (2014).

[7] T. Niemczyk, F. Deppe, H. Huebl, E. P. Menzel, F. Hocke, M. J. Schwarz, J. J. Garcia-Ripoll, D. Zueco, T. Hummer, E. Solano, A. Marx, and R. Gross, Nat. Phys. 6, 772 (2010).
[8] P. Forn-Díaz, J. Lisenfeld, D. Marcos, J. J. García-Ripoll, E. Solano, C. J. P. M. Harmans, and J. E. Mooij, Phys. Rev. Lett. 105, 237001 (2010)

[9] E. P. Menzel, R. Di Candia, F. Deppe, P. Eder, L. Zhong, M. Ihmig, M. Haeberlein, A. Baust, E. Hoffmann, D. Ballester, K. Inomata, T. Yamamoto, Y. Nakamura, E. Solano, A. Marx, and R. Gross, Phys. Rev. Lett. 109, 250502 (2012).

[10] L. Zhong, E. P. Menzel, R. D. Candia, P. Eder, M. Ihmig, A. Baust, M. Haeberlein, E. Hoffmann, K. Inomata, T. Yamamoto, Y. Nakamura, E. Solano, F. Deppe, A. Marx, and R. Gross, New J. Phys. 15, 125013 (2013).

[11] L. Steffen, Y. Salathe, M. Oppliger, P. Kurpiers, M. Baur, C. Lang, C. Eichler, G. Puebla-Hellmann, A. Fedorov, and A. Wallraff, Nature (London) 500, 319 (2013).

[12] P. Roushan, C. Neill, Y. Chen, M. Kolodrubetz, C. Quintana, N. Leung, M. Fang, R. Barends, B. Campbell, Z. Chen, B. Chiaro, A. Dunsworth, E. Jeffrey, J. Kelly, A. Megrant, J. Mutus, P. J. J. O’Malley, D. Sank, A. Vainsencher, J. Wenner, T. White, A. Polkovnikov, A. N. Cleland, and J. M. Martinis, Nature (London) 515, 241 (2014).

[13] I. Rabi, Phys. Rev. 49, 324 (1936).

[14] D. Braak, Phys. Rev. Lett. 107, 100401 (2011). 
[15] G. Romero, D. Ballester, Y. M. Wang, V. Scarani, and E. Solano, Phys. Rev. Lett. 108, 120501 (2012).

[16] I. Lizuain, J. Casanova, J. J. García-Ripoll, J. G. Muga, and E. Solano, Phys. Rev. A 81, 062131 (2010).

[17] S. Felicetti, G. Romero, D. Rossini, R. Fazio, and E. Solano, Phys. Rev. A 89, 013853 (2014).

[18] D. Ballester, G. Romero, J. J. García-Ripoll, F. Deppe, and E. Solano, Phys. Rev. X 2, 021007 (2012).

[19] T. Niemczyk, F. Deppe, M. Mariantoni, E. P. Menzel, E. Hoffmann, G. Wild, L. Eggenstein, A. Marx, and R. Gross, Supercond. Sci. Technol. 22, 034009 (2009).

[20] E. Hoffmann, Ph.D. thesis, Garching, 2013.

[21] A. Baust, E. Hoffmann, M. Haeberlein, M. J. Schwarz, P. Eder, J. Goetz, F. Wulschner, E. Xie, L. Zhong, F. Quijandría, B. Peropadre, D. Zueco, J.-J. García Ripoll, E. Solano, K. Fedorov, E. P. Menzel, F. Deppe, A. Marx, and R. Gross, Phys. Rev. B 91, 014515 (2015).

[22] M. Mariantoni, F. Deppe, A. Marx, R. Gross, F. K. Wilhelm, and E. Solano, Phys. Rev. B 78, 104508 (2008).

[23] M. Wallquist, V. S. Shumeiko, and G. Wendin, Phys. Rev. B 74, 224506 (2006).
[24] M. Sandberg, C. M. Wilson, F. Persson, T. Bauch, G. Johansson, V. Shumeiko, T. Duty, and P. Delsing, Appl. Phys. Lett. 92, 203501 (2008).

[25] A. Görür and C. Karpuz, Microw. Opt. Technol. Lett. 22, 123 (1999).

[26] T. P. Orlando, J. E. Mooij, L. Tian, C. H. van der Wal, L. S. Levitov, S. Lloyd, and J. J. Mazo, Phys. Rev. B 60, 15398 (1999).

[27] F. Terman, Radio Engineers Handbook (McGraw-Hill, New York, 1945).

[28] M. J. Schwarz, J. Goetz, Z. Jiang, T. Niemczyk, F. Deppe, A. Marx, and R. Gross, New J. Phys. 15, 045001 (2013).

[29] M. J. Schwarz, Ph.D. thesis, Garching, 2013.

[30] J. Casanova, G. Romero, I. Lizuain, J. J. García-Ripoll, and E. Solano, Phys. Rev. Lett. 105, 263603 (2010).

[31] A. Ridolfo, M. Leib, S. Savasta, and M. J. Hartmann, Phys. Rev. Lett. 109, 193602 (2012).

[32] S. De Liberato, D. Gerace, I. Carusotto, and C. Ciuti, Phys. Rev. A 80, 053810 (2009).

[33] J. M. Fink, M. Baur, R. Bianchetti, S. Filipp, M. Göppl, P. J. Leek, L. Steffen, A. Blais, and A. Wallraff, Phys. Scr. 2009, 014013 (2009). 